\title{
CLOSE-TO-CONVEXITY OF NORMALIZED WRIGHT FUNCTIONS
}

\author{
(NORMALIZE WRIGHT FONKSIONLARININ KONVEKSE-YAKINLIĞI)
}

\author{
Nizami MUSTAFA ${ }^{1}$
}

\begin{abstract}
In this paper, a new subclass $K(\alpha, \beta), \alpha, \beta \in[0,1)$ of analytic functions in the open unit disk is introduced. The purpose of the present paper is to investigate some characterizations for the normalized Wright functions to be in the subclass $K(\alpha, \beta), \alpha, \beta \in[0,1)$. In this study, various sufficient conditions for the normalized Wright functions to be in this class are also obtained.
\end{abstract}

Keywords: Wright function, Starlike function, Convex function, Close-To-Convex function

\section{$\ddot{O} Z$}

Bu makalede açık birim diskte analitik fonksiyonların $K(\alpha, \beta), \alpha, \beta \in[0,1)$ yeni bir alt sınıf tanımlandl. Makalenin amacı, normalize Wright fonksiyonlarının analitik fonksiyonların $K(\alpha, \beta), \alpha, \beta \in[0,1)$ alt sınıfina ait olması içinbazı karakterizasyonları araştırmaktır. Bu çalışmada normalize Wright fonksiyonlarının bu sınıfa ait olması için çeşitli yeterli koşullar da elde edilir.

Anahtar kelimeler: Wright fonksiyon, Yıldızll fonksiyon, Konveks fonksiyon, Konvekse-yakın fonksiyon

\footnotetext{
${ }^{1}$ Department of Mathematics, Faculty of Science and Letters, Kafkas University, TR-36100 Kars, Turkey, email: nizamimustafa@gmail.com
} 


\section{INTRODUCTION}

It is well known that the special functions play an important role in geometric function theory. It is also well known that the application area of the special functions is not limited to the theory of geometric functions. These functions are also wide range of applications in many problems as well as in other branches of mathematics and applied sciences.

In this paper, we will examine Wright function, which is defined by the infinite series

$$
W_{\lambda, \mu}(z)=\sum_{n=0}^{\infty} \frac{1}{\Gamma(\lambda n+\mu)} \frac{z^{n}}{n !}, \lambda>-1, \mu, z \in \square
$$

This series is absolutely convergent in $\square$, when $\lambda>-1$ and absolutely convergent in open unit disk for $\lambda=-1$. Furthermore, for $\lambda>-1$, the Wright function $W_{\lambda, \mu}(z)$ is an entire function. The Wright function was introduced by Wright [1] and has appeared for the first time in the case $\lambda>0$ in connection with his investigations in the asymptotic theory of partitions. Later on, it has found many other applications, first of all, in the Mikusinski operational calculus and in the theory of integral transforms of Hankel type. Furthermore, extending the methods of Lie groups in partial differential equations to the partial differential equations of fractional order, it was shown that some of the group-invariant solutions of these equations can be given in terms of the Wright function.

Note that, the Wright function generalizes various functions like Array function, Whittaker function, (Wright-type) entire auxiliary functions, etc. For the details, we refer to [2]. Recently, Wright function has appeared in papers related to partial differential equations of fractional order, it was found that the corresponding Green functions can be represented in terms of the Wright function [3,4]. A series of paper are devoted to the application of the Wright functions in partial differential equation of fractional order extending the classical diffusion and wave equations. Mainardi has obtained the result for a fractional diffusion wave equation in terms of the fractional Green function involving the Wright function [5]. The scale-variant solutions of some partial differential equations of fractional order were obtained in terms of special cases of the generalized Wright function by Luchko and Gorenflo [6].

If $\lambda$ is a positive rational number, then the Wright function $W_{\lambda, \mu}(z)$ can be represented in terms of the more familiar generalized hypergeometric functions (see [2, section 2.1]). In particular, when $\lambda=1$ and $\mu=p+1$, the functions $W_{1, p+1}\left(-z^{2} / 4\right)$ are expressed in terms of the Bessel functions $J_{p}(z)$, given as follows:

$J_{p}(z)=\left(\frac{z}{2}\right)^{p} W_{1, p+1}\left(-\frac{z^{2}}{4}\right)=\sum_{n=0}^{\infty} \frac{(-1)^{n}}{\Gamma(n+p+1)} \frac{(z / 2)^{2 n+p}}{n !}$.

Furthermore, the function $W_{\lambda, p+1}(-z) \equiv J_{p}^{\lambda}(z)(\lambda>0, p>-1)$ is known as the generalized Bessel function (misnamed also as the Bessel-Maitland function). 
Recently, Prajapat [7] investigated some geometric properties of the normalized Wright functions, and has obtained interesting results.

\section{PRELIMINARIES}

Let $A$ be the class of analytic in the open unit disk $U=\{z \in \square:|z|<1\}$ functions $f(z)$, normalized by $f(0)=0=f^{\prime}(0)-1$ of the form

$$
f(z)=z+a_{2} z^{2}+a_{3} z^{3}+\cdots+a_{n} z^{n}+\cdots=z+\sum_{n=2}^{\infty} a_{n} z^{n} .
$$

Also, let $S^{*}(\alpha), C(\alpha)$ and $K(\alpha)$ denote the subclasses of $A$ consisting of functions which are, respectively, starlike, convex and close-to-convex with respect to starlike function $g(z)$ (need not be normalized) of order $\alpha(\alpha \in[0,1))$ in the open unit disk $U$. Thus, we have (see for details, $[8,9]$, also [10])

$$
\begin{aligned}
& S^{*}(\alpha)=\left\{f \in A: \operatorname{Re}\left(\frac{z f^{\prime}(z)}{f(z)}\right)>\alpha, z \in U\right\}, \alpha \in[0,1), \\
& C(\alpha)=\left\{f \in A: \operatorname{Re}\left(1+\frac{z f^{\prime \prime}(z)}{f^{\prime}(z)}\right)>\alpha, z \in U\right\}, \alpha \in[0,1)
\end{aligned}
$$

and

$$
K(\alpha)=\left\{f \in A: \operatorname{Re}\left(\frac{z f^{\prime}(z)}{g(z)}\right)>\alpha, z \in U, g \in S^{*}\right\}, \alpha \in[0,1) .
$$

In the special cases, $S^{*}=S^{*}(0), C=C(0)$ and $K=K(0)$, are, respectively, starlike, convex and close-to-convex functions in $U$. It is well known that close-to-convex functions are univalent in $U$, but not necessarily the converse. It is easy to verify that $C \subset S^{*} \subset K$. For details on these classes, one could refer to the monograph by Goodman [11].

An interesting generalization of the function classes $S^{*}(\alpha), \quad C(\alpha)$ and $K(\alpha)$ are provided by the classes $S^{*}(\alpha, \beta), C(\alpha, \beta)$ and $K(\alpha, \beta)$ of functions $f \in A$, which satisfies the following conditions:

$$
\begin{aligned}
& S^{*}(\alpha, \beta)=\left\{f \in A: \operatorname{Re}\left(\frac{z f^{\prime}(z)+\beta z^{2} f^{\prime \prime}(z)}{f(z)}\right)>\alpha, z \in U\right\}, \alpha, \beta \in[0,1), \\
& C(\alpha, \beta)=\left\{f \in A: \operatorname{Re}\left(\frac{\left[z f^{\prime}(z)+\beta z^{2} f^{\prime \prime}(z)\right]^{\prime}}{f^{\prime}(z)}\right)>\alpha, z \in U\right\}, \alpha, \beta \in[0,1)
\end{aligned}
$$


and

$$
K(\alpha, \beta)=\left\{f \in A: \operatorname{Re}\left(\frac{z f^{\prime}(z)+\beta z^{2} f^{\prime \prime}(z)}{g(z)}\right)>\alpha, z \in U, g \in S^{*}(\alpha, \beta)\right\}, \alpha, \beta \in[0,1)
$$

with respect to function $g(z)$ (need not be normalized), respectively.

Note 2.1. The class $K(\alpha, \beta), \alpha, \beta \in[0,1)$ is the first time introduced and examined in this paper.Clearly, for $\beta=0$, we have $K(\alpha, 0)=K(\alpha)$.

The Wright function $W_{\lambda, \mu}(z)$ defined by (1) does not belong to the class $A$. Thus, it is natural to consider the following two kinds of normalization of the Wright function:

$W_{\lambda, \mu}^{(1)}(z):=\Gamma(\mu) \mathrm{z} W_{\lambda, \mu}(z)=\sum_{n=0}^{\infty} \frac{\Gamma(\mu)}{\Gamma(\lambda n+\mu)} \frac{z^{n+1}}{n !}, \lambda>-1, \mu>0, z \in U$

and

$W_{\lambda, \mu}^{(2)}(z):=\Gamma(\lambda+\mu)\left[W_{\lambda, \mu}(z)-\frac{1}{\Gamma(\mu)}\right]=\sum_{n=0}^{\infty} \frac{\Gamma(\lambda+\mu)}{\Gamma(\lambda n+\lambda+\mu)} \frac{z^{n+1}}{(n+1) !}, \lambda>-1, \lambda+\mu>0, z \in U$.

Easily, we write

$$
W_{\lambda, \mu}^{(1)}(z)=z+\sum_{n=2}^{\infty} \frac{\Gamma(\mu)}{\Gamma(\lambda(n-1)+\mu)} \frac{z^{n}}{(n-1) !}, \lambda>-1, \mu>0, z \in U
$$

$$
W_{\lambda, \mu}^{(2)}(z)=z+\sum_{n=2}^{\infty} \frac{\Gamma(\lambda+\mu)}{\Gamma(\lambda(n-1)+\lambda+\mu)} \frac{z^{n}}{n !}, \lambda>-1, \lambda+\mu>0, z \in U
$$

Furthermore, we observe that $W_{\lambda, \mu}^{(1)}$ and $W_{\lambda, \mu}^{(2)}$ are satisfying the following relations:

$$
\begin{aligned}
& \lambda z\left(W_{\lambda, \mu}^{(1)}(z)\right)^{\prime}=(\mu-1) W_{\lambda, \mu-1}^{(1)}(z)+(\lambda-\mu+1) W_{\lambda, \mu}^{(1)}(z), \\
& \lambda z\left(W_{\lambda, \mu}^{(2)}(z)\right)^{\prime}=(\lambda+\mu-1) W_{\lambda, \mu-1}^{(2)}(z)+(1-\mu) W_{\lambda, \mu}^{(2)}(z), \\
& z\left(W_{\lambda, \mu}^{(2)}(z)\right)^{\prime}=W_{\lambda, \lambda+\mu}^{(1)}(z) \text { and } V_{\lambda, \mu}^{\prime}(z)=\frac{\Gamma(\mu)}{\Gamma(\lambda+\mu)} V_{\lambda, \lambda+\mu}(z), \\
& \text { where } V_{\lambda, \mu}(z)=\frac{W_{\lambda, \mu}^{(1)}(z)}{z} .
\end{aligned}
$$

Note that 


$$
-W_{1, p+1}^{(1)}(-z)=\bar{J}_{p}(z):=\Gamma(p+1) \mathrm{z}^{1-p / 2} J_{p}(2 \sqrt{z}), p>-1 .
$$

Here, $\bar{J}_{p}(z)$ is the normalized Bessel function.

In this paper, we give sufficient conditions for the parameters of the normalized Wright functions to be in the class $K(\alpha, \beta)$.

The following lemma will be required.

Lemma 2.1. [12] A function $f \in A$ belongs to the class $S^{*}(\alpha, \beta)$ if

$$
\sum_{n=2}^{\infty}(n+\beta n(n-1)-\alpha)\left|a_{n}\right| \leq 1-\alpha .
$$

\section{SUFFICIENT CONDITIONS FOR THE CLASS $K(\alpha, \beta)$}

In this section, we will give sufficient conditions for the parameters of the normalized Wright functions to be in the class $K(\alpha, \beta)$.

Theorem 3.1. Let $\lambda \geq 1, \mu \geq \mu_{0}=0.462$ and the following condition is satisfied:

$$
(1-\alpha)(\mu+1) \mu-\left[(\mu+1)^{2}+(1+2 \beta)(\mu+1)+\beta\right] e^{\frac{1}{\mu+1}}+(\mu+1)^{2}>0 .
$$

Then, normalized Wright function $W_{\lambda, \mu}^{(1)}(z)$ belongs to the class $K(\alpha, \beta)$.

Proof. By the definition, we need to show that $\operatorname{Re}\left\{\left(W_{\lambda, \mu}^{(1)}(z)\right)^{\prime}+\beta z\left(W_{\lambda, \mu}^{(1)}(z)\right)^{\prime \prime}\right\}>\alpha, z \in U$, this can be shown by proving

$$
\left|\left(W_{\lambda, \mu}^{(1)}(z)\right)^{\prime}+\beta z\left(W_{\lambda, \mu}^{(1)}(z)\right)^{\prime \prime}-1\right|<1-\alpha, z \in U
$$

By simple computation, we obtain

$$
\begin{aligned}
& \left|\left(W_{\lambda, \mu}^{(1)}(z)\right)^{\prime}+\beta z\left(W_{\lambda, \mu}^{(1)}(z)\right)^{\prime \prime}-1\right|=\left|\sum_{n=2}^{\infty} \frac{n+n(n-1) \beta}{(n-1) !} \frac{\Gamma(\mu)}{\Gamma(\lambda(n-1)+\mu)} z^{n-1}\right| \\
& \leq \sum_{n=2}^{\infty} \frac{\beta n^{2}+(1-\beta) n}{(n-1) !} \frac{\Gamma(\mu)}{\Gamma(\lambda(n-1)+\mu)} .
\end{aligned}
$$

Let

$$
L_{1}(\lambda, \mu ; \beta)=\sum_{n=2}^{\infty} \frac{\beta n^{2}+(1-\beta) n}{(n-1) !} \frac{\Gamma(\mu)}{\Gamma(\lambda(n-1)+\mu)} .
$$


Setting $n^{2}=(n-1)(n-2)+3(n-1)+1, n=(n-1)+1$ and by simple computation, we write

$$
\begin{aligned}
& L_{1}(\lambda, \mu ; \beta)=\sum_{n=3}^{\infty} \frac{\beta}{(n-3) !} \frac{\Gamma(\mu)}{\Gamma(\lambda(n-1)+\mu)}+\sum_{n=2}^{\infty} \frac{1+2 \beta}{(n-2) !} \frac{\Gamma(\mu)}{\Gamma(\lambda(n-1)+\mu)} \\
& +\sum_{n=2}^{\infty} \frac{1}{(n-1) !} \frac{\Gamma(\mu)}{\Gamma(\lambda(n-1)+\mu)} .
\end{aligned}
$$

Under the hypothesis $\lambda \geq 1$, the inequality $\Gamma(n-1+\mu) \leq \Gamma(\lambda(n-1)+\mu)$ for $n \in \square$ holds, which is equivalent to

$$
\frac{\Gamma(\mu)}{\Gamma(\lambda(n-1)+\mu)} \leq \frac{1}{(\mu)_{n-1}}, n \in \square
$$

Here, $(\mu)_{n}=\frac{\Gamma(n+\mu)}{\Gamma(\mu)}=\mu(\mu+1)(\mu+2) \cdots(\mu+n-1),(\mu)_{0}=1$ is Pochhammer (or Appell) symbol, defined in terms of Euler gamma function. Using (9), we get

$$
L_{1}(\lambda, \mu ; \beta) \leq \sum_{n=3}^{\infty} \frac{\beta}{(n-3) !} \frac{1}{(\mu)_{n-1}}+\sum_{n=2}^{\infty} \frac{1+2 \beta}{(n-2) !} \frac{1}{(\mu)_{n-1}}+\sum_{n=2}^{\infty} \frac{1}{(n-1) !} \frac{1}{(\mu)_{n-1}} .
$$

Further, the inequality

$$
(\mu)_{n-1}=\mu(\mu+1)(\mu+2) \cdots(\mu+n-2) \geq \mu(\mu+1)^{n-2}, n \in \square
$$

holds, which is equivalent to $1 /(\mu)_{n-1} \leq 1 / \mu(\mu+1)^{n-2}, n \in \square$.

Using (10), we obtain

$$
\begin{aligned}
& L_{1}(\lambda, \mu ; \beta) \leq \sum_{n=3}^{\infty} \frac{\beta}{(n-3) !} \frac{1}{\mu(\mu+1)^{n-2}}+\sum_{n=2}^{\infty} \frac{1+2 \beta}{(n-2) !} \frac{1}{\mu(\mu+1)^{n-2}}+\sum_{n=2}^{\infty} \frac{1}{(n-1) !} \frac{1}{\mu(\mu+1)^{n-2}} \\
& =\left[\frac{\beta}{\mu(\mu+1)}+\frac{1+2 \beta}{\mu}+\frac{\mu+1}{\mu}\right] e^{\frac{1}{\mu+1}}-\frac{\mu+1}{\mu}<1-\alpha,
\end{aligned}
$$

which is equivalent to

$$
(1-\alpha)(\mu+1) \mu-\left[(\mu+1)^{2}+(1+2 \beta)(\mu+1)+\beta\right] e^{\frac{1}{\mu+1}}+(\mu+1)^{2}>0 .
$$

Thus, the proof of Theorem 3.1 is complete.

By setting $\beta=0$ in Theorem 3.1 and using the relationship $K(\alpha, 0)=K(\alpha)$, we arrive at the following corollary. 
Corollary 3.1. The normalized Wright function $W_{\lambda, \mu}^{(1)}(z)$ belongs to the class $K(\alpha)$ if $\lambda \geq 1, \mu \geq \mu_{0}=0.462$ and the following condition is satisfied:

$$
(2-\alpha) \mu-(\mu+2) e^{\frac{1}{\mu+1}}+1>0 .
$$

By taking $\alpha=0$ in Corollary 3.1 and using the relationship $K(0)=K$, we obtain the following corollary.

Corollary 3.2. Let $\lambda \geq 1$ and $\mu>x_{0}$, where $x_{0} \cong 2.4898$ is the root of the equation

$2 x-(x+2) e^{\frac{1}{x+1}}+1=0$,

then $W_{\lambda, \mu}^{(1)} \in K$.

Proof. Let $\varphi(x)=2 x-(x+2) e^{1 /(x+1)}+1, x>0$. By simple computation, we obtain $\varphi^{\prime}(x)=\frac{x+2}{(x+1)^{2}} e^{1 /(x+1)}+2-e^{1 /(x+1)}$.

Easily, we see that $\varphi^{\prime}(x)>0$ for each $x>0$. Thus, function $\varphi(x)$ is an increasing function. Hence, $2 \mu-(\mu+2) e^{1 /(\mu+1)}+1>0$ for every $\mu>x_{0}$. Here, $x_{0} \cong 2.4898$ is the root of the equation

$2 x-(x+2) e^{\frac{1}{x+1}}+1=0$.

Thus, the proof of Corollary 3.2 is complete.

Theorem 3.2. Let $\lambda \geq 1, \lambda+\mu>0$ and the following condition is satisfied:

$(1-\alpha)(\lambda+\mu)-\left(e^{\frac{1}{\lambda+\mu+1}}-1\right)(\lambda+\mu+1)-\beta e^{\frac{1}{\lambda+\mu+1}}>0$.

Then, normalized Wright function $W_{\lambda, \mu}^{(2)}(z)$ belongs to the class $K(\alpha, \beta)$.

Proof. By the definition, we need to show that $\operatorname{Re}\left\{\left(W_{\lambda, \mu}^{(2)}(z)\right)^{\prime}+\beta z\left(W_{\lambda, \mu}^{(2)}(z)\right)^{\prime \prime}\right\}>\alpha, z \in U$, this can be shown by proving $\left|\left(W_{\lambda, \mu}^{(2)}(z)\right)^{\prime}+\beta z\left(W_{\lambda, \mu}^{(2)}(z)\right)^{\prime \prime}-1\right|<1-\alpha, z \in U$

Since $\left(W_{\lambda, \mu}^{(2)}(z)\right)^{\prime}=\frac{W_{\lambda, \lambda+\mu}^{(1)}(z)}{z}$, by simple computation, we write 


$$
\begin{aligned}
& \left|\left(W_{\lambda, \mu}^{(2)}(z)\right)^{\prime}+\beta z\left(W_{\lambda, \mu}^{(2)}(z)\right)^{\prime \prime}-1\right|=\left|\frac{W_{\lambda, \lambda+\mu}^{(1)}(z)}{z}-1+\beta\left[\left(W_{\lambda, \lambda+\mu}^{(1)}(z)\right)^{\prime}-\frac{W_{\lambda, \lambda+\mu}^{(1)}(z)}{z}\right]\right| \\
& =\left|\sum_{n=2}^{\infty}\left\{\frac{\beta}{(n-2) !}+\frac{1}{(n-1) !}\right\} \frac{\Gamma(\lambda+\mu)}{\Gamma(\lambda(n-1)+\lambda+\mu)} z^{n-1}\right| \leq \sum_{n=2}^{\infty}\left\{\frac{\beta}{(n-2) !}+\frac{1}{(n-1) !}\right\} \frac{\Gamma(\lambda+\mu)}{\Gamma(\lambda(n-1)+\lambda+\mu)} .
\end{aligned}
$$

Under the hypothesis $\lambda \geq 1$, using (9) and (10) with $\mu \equiv \lambda+\mu$, we obtain

$$
\begin{aligned}
& \left|\left(W_{\lambda, \mu}^{(2)}(z)\right)^{\prime}+\beta z\left(W_{\lambda, \mu}^{(2)}(z)\right)^{\prime \prime}-1\right| \leq \sum_{n=2}^{\infty}\left\{\frac{\beta}{(n-2) !}+\frac{1}{(n-1) !}\right\} \frac{1}{(\lambda+\mu)(\lambda+\mu+1)^{n-2}} \\
& =\frac{\lambda+\mu+\beta+1}{\lambda+\mu} e^{\frac{1}{\lambda+\mu+1}}-\frac{\lambda+\mu+1}{\lambda+\mu} .
\end{aligned}
$$

Thus,

$$
\frac{\lambda+\mu+\beta+1}{\lambda+\mu} e^{\frac{1}{\lambda+\mu+1}}-\frac{\lambda+\mu+1}{\lambda+\mu}<1-\alpha,
$$

which is equivalent to

$$
(1-\alpha)(\lambda+\mu)-\left(e^{\frac{1}{\lambda+\mu+1}}-1\right)(\lambda+\mu+1)-\beta e^{\frac{1}{\lambda+\mu+1}}>0 .
$$

Thus, the proof of Theorem 3.2 is complete.

By setting $\beta=0$ in Theorem 3.2 and using the relationship $K(\alpha, 0)=K(\alpha)$, we arrive at the following corollary.

Corollary 3.3. The normalized Wright function $W_{\lambda, \mu}^{(2)}(z)$ belongs to the class $K(\alpha)$ if $\lambda \geq 1$, $\lambda+\mu>0$ and the following condition is satisfied:

$\left(2-\alpha-e^{\frac{1}{\lambda+\mu+1}}\right)(\lambda+\mu)-e^{\frac{1}{\lambda+\mu+1}}+1>0$.

By taking $\alpha=0$ in Corollary 3.3 and using the relationship $K(0)=K$, we obtain the following corollary.

Corollary 3.4. Let $\lambda \geq 1, \lambda+\mu>0$ and $\lambda+\mu>x_{1}$, where $x_{1} \cong 1.2581$ is the root of the equation

$$
2 x-(x+1) e^{\frac{1}{x+1}}+1=0,
$$

then $W_{\lambda, \mu}^{(2)} \in K$. 
Proof. Let $h(x)=2 x-(x+1) e^{1 /(x+1)}+1, x>0$. By simple computation, we obtain $h^{\prime}(x)=2-\frac{x}{x+1} e^{\frac{1}{x+1}}$

It can be seen that $h^{\prime}(x)>0$ for each $x>0$. Hence, function $h(x)$ is an increasing function. So $2(\lambda+\mu)-(\lambda+\mu+1) e^{\frac{1}{\lambda+\mu+1}}+1>0$ for every $\lambda+\mu>x_{1}$. Here, $x_{1} \cong 1.2581$ is root of the equation

$2 x-(x+1) e^{\frac{1}{x+1}}+1=0$.

Thus, the proof of Corollary 3.4 is complete.

Theorem 3.3. Let $\lambda \geq 1$ and the following condition is satisfied:

$\left\{(1-\alpha)\left[(2 p+3)-(p+2) e^{\frac{1}{p+2}}\right]-\left[2\left(e^{\frac{1}{p+2}}-1\right)(p+2)+(1+2 \beta) e^{\frac{1}{p+2}}\right]\right\}(p+2)-\beta e^{\frac{1}{p+2}}>0$.

Then, normalized Wright function $W_{\lambda, p+1}^{(1)}(z)$ belongs to the class $K(\alpha, \beta)$ with respect to $\bar{J}_{p}(z)$ in $U$.

Proof. By the definition, we need to show that

$\operatorname{Re}\left\{\frac{z\left(W_{\lambda, p+1}^{(1)}(z)\right)^{\prime}+\beta z^{2}\left(W_{\lambda, p+1}^{(1)}(z)\right)^{\prime \prime}}{\bar{J}_{p}(z)}\right\}>\alpha, z \in U$

this can be shown by proving

$\left|1-\frac{z\left(W_{\lambda, p+1}^{(1)}(z)\right)^{\prime}+\beta z^{2}\left(W_{\lambda, p+1}^{(1)}(z)\right)^{\prime \prime}}{\bar{J}_{p}(z)}\right|<1-\alpha, z \in U$

For $z \in U$, by simple computation, we obtain

$$
\begin{aligned}
& \left|\left(W_{\lambda, p+1}^{(1)}(z)\right)^{\prime}-\frac{\bar{J}_{p}(z)}{z}+\beta z\left(W_{\lambda, p+1}^{(1)}(z)\right)^{\prime \prime}\right| \leq \sum_{n=1}^{\infty} \frac{\Gamma(p+1)}{n !}\left|\left[\frac{n+1}{\Gamma(\lambda n+p+1)}-\frac{(-1)^{n}}{\Gamma(n+p+1)}\right] z^{n}\right| \\
& +\beta \sum_{n=1}^{\infty} \frac{\Gamma(p+1)}{\Gamma(\lambda n+p+1)} \frac{n(n+1)}{n !} .
\end{aligned}
$$

Under the hypothesis $\lambda \geq 1$, the inequality $\Gamma(n+p+1) \leq \Gamma(\lambda n+p+1)$ for each $n \in \square$ holds, which is equivalent to 


$$
\frac{\Gamma(p+1)}{\Gamma(\lambda n+p+1)} \leq \frac{\Gamma(p+1)}{\Gamma(n+p+1)}=\frac{1}{(p+1)_{n}}, n \in \square,
$$

where $(p+1)_{n}=\frac{\Gamma(n+p+1)}{\Gamma(p+1)}=(p+1)(p+2) \ldots(p+n)$.

Further, the following inequality holds

$$
\frac{1}{(p+1)_{n}} \leq \frac{1}{(p+1)(p+2)^{n-1}}, n \in \sqcup .
$$

Using (12) and (13), from (11), we obtain

$$
\begin{aligned}
& \left|\left(W_{\lambda, p+1}^{(1)}(z)\right)^{\prime}-\frac{\bar{J}_{p}(z)}{z}+\beta z\left(W_{\lambda, p+1}^{(1)}(z)\right)^{\prime \prime}\right| \leq \sum_{n=1}^{\infty} \frac{n+2}{n !} \frac{1}{(p+1)(p+2)^{n-1}}+\beta \sum_{n=1}^{\infty} \frac{n(n+1)}{n !} \frac{1}{(p+1)(p+2)^{n-1}} \\
& =2 \sum_{n=1}^{\infty} \frac{1}{n !} \frac{1}{(p+1)(p+2)^{n-1}}+(1+2 \beta) \sum_{n=1}^{\infty} \frac{1}{(n-1) !} \frac{1}{(p+1)(p+2)^{n-1}}+\beta \sum_{n=2}^{\infty} \frac{1}{(n-2) !} \frac{1}{(p+1)(p+2)^{n-1}} \\
& =\frac{2(p+2)}{p+1}\left(e^{\frac{1}{p+2}}-1\right)+\frac{1+2 \beta}{p+1} e^{\frac{1}{p+2}}+\frac{\beta}{(p+1)(p+2)} e^{\frac{1}{p+2}}\left\{2\left(e^{\frac{1}{p+2}}-1\right)(p+2)^{2}+(1+2 \beta)(p+2) e^{\frac{1}{p+2}}+\beta e^{\frac{1}{p+2}}\right\} .
\end{aligned}
$$

Similarly, we obtain

$$
\left|\frac{\bar{J}_{p}(z)}{z}\right| \geq 1-\sum_{n=1}^{\infty} \frac{\Gamma(p+1)}{\Gamma(n+p+1)} \frac{1}{n !} \geq 1-\sum_{n=1}^{\infty} \frac{1}{n !} \frac{1}{(p+1)(p+2)^{n-1}}=2-\frac{1}{p+1}\left[(p+2) e^{\frac{1}{p+2}}-1\right] .
$$

From (14) and (15), we obtain

$$
\left|1-\frac{z\left(W_{\lambda, p+1}^{(1)}(z)\right)^{\prime}+\beta z^{2}\left(W_{\lambda, p+1}^{(1)}(z)\right)^{\prime \prime}}{\bar{J}_{p}(z)}\right| \leq \frac{2\left(e^{\frac{1}{p+2}}-1\right)(p+2)^{2}+(1+2 \beta)(p+2) e^{\frac{1}{p+2}}+\beta e^{\frac{1}{p+2}}}{\left[(2 p+3)-(p+2) e^{\frac{1}{p+2}}\right](p+2)} .
$$

Thus, we get

$$
\left\{(1-\alpha)\left[(2 p+3)-(p+2) e^{\frac{1}{p+2}}\right]-\left[2\left(e^{\frac{1}{p+2}}-1\right)(p+2)+(1+2 \beta) e^{\frac{1}{p+2}}\right]\right\}(p+2)-\beta e^{\frac{1}{p+2}}>0
$$

This shows that 
$\operatorname{Re}\left\{\frac{z\left(W_{\lambda, p+1}^{(1)}(z)\right)^{\prime}+\beta z^{2}\left(W_{\lambda, p+1}^{(1)}(z)\right)^{\prime \prime}}{\bar{J}_{p}(z)}\right\}>\alpha$

if condition (16) is satisfied.

For the completion of the proof of Theorem 3.3, we need to show that function $\bar{J}_{p}(z)$ belongs to the class $S^{*}(\alpha, \beta)$ if condition (16) is satisfied.

From (8), we write

$\bar{J}_{p}(z)=-W_{1, p+1}^{(1)}(-z)=z+\sum_{n=2}^{\infty} \frac{\Gamma(p+1)}{\Gamma(n+p)} \frac{(-1)^{n+1}}{(n-1) !} z^{n}$

According to the Lemma 2.1, function $\bar{J}_{p}(z)$ belongs to the class $S^{*}(\alpha, \beta)$ if the following condition is satisfied:

$\sum_{n=2}^{\infty}\left[n^{2} \beta+n(1-\beta)-\alpha\right] \frac{\Gamma(p+1)}{\Gamma(n+p)} \frac{1}{(n-1) !} \leq 1-\alpha$.

Let

$L_{2}(p ; \alpha, \beta)=\sum_{n=2}^{\infty}\left[n^{2} \beta+n(1-\beta)-\alpha\right] \frac{\Gamma(p+1)}{\Gamma(n+p)} \frac{1}{(n-1) !}$.

Using similar calculations to get $L_{1}(\lambda, \mu ; \beta)$, we write

$L_{2}(p ; \alpha, \beta)=\sum_{n=3}^{\infty} \frac{\beta}{(n-3) !} \frac{\Gamma(p+1)}{\Gamma(n+p)}+\sum_{n=2}^{\infty} \frac{1+2 \beta}{(n-2) !} \frac{\Gamma(p+1)}{\Gamma(n+p)}+\sum_{n=2}^{\infty} \frac{1-\alpha}{(n-1) !} \frac{\Gamma(p+1)}{\Gamma(n+p)}$.

The following equality is clear

$\frac{\Gamma(p+1)}{\Gamma(n+p)}=\frac{1}{(p+1)_{n-1}}, n \in \square$.

Using (17) and (10), with $\mu \equiv p+1$, we get

$L_{2}(p ; \alpha, \beta) \leq \sum_{n=3}^{\infty} \frac{\beta}{(n-3) !} \frac{1}{(p+1)(p+2)^{n-2}}+\sum_{n=2}^{\infty} \frac{1+2 \beta}{(n-2) !} \frac{1}{(p+1)(p+2)^{n-2}}$

$+\sum_{n=2}^{\infty} \frac{1-\alpha}{(n-1) !} \frac{1}{(p+1)(p+2)^{n-2}}=\left[\frac{\beta}{(p+1)(p+2)}+\frac{1+2 \beta}{p+1}+\frac{(1-\alpha)(p+2)}{p+1}\right] e^{\frac{1}{p+2}}$

$-\frac{(1-\alpha)(p+2)}{p+1} \leq 1-\alpha$,

which is equivalent to 


$$
\left\{(1-\alpha)\left[(2 p+3)-(p+2) e^{\frac{1}{p+2}}\right]-(1+2 \beta) e^{\frac{1}{p+2}}\right\}(p+2)-\beta e^{\frac{1}{p+2}} \geq 0 .
$$

Thus, condition (18) is a sufficient condition for that function $\bar{J}_{p}(z)$ is in the class $S^{*}(\alpha, \beta)$. Also, from (16) the following condition is satisfied:

$$
\left\{(1-\alpha)\left[(2 p+3)-(p+2) e^{\frac{1}{p+2}}\right]-(1+2 \beta) e^{\frac{1}{p+2}}\right\}(p+2)-\beta e^{\frac{1}{p+2}}>2\left(e^{\frac{1}{p+2}}-1\right)(p+2)^{2}
$$

But since $e^{\frac{1}{p+2}}>1$ for $p>-1$, condition (18) is also satisfied. Thus, from the hypothesis of theorem, $\bar{J}_{p} \in S^{*}(\alpha, \beta)$.

The proof is complete.

By setting $\beta=0$ in Theorem 3.3 and using the relationship $K(\alpha, 0)=K(\alpha)$, we arrive at the following corollary.

Corollary 3.5. If $\lambda \geq 1$ and the following condition is satisfied:

$(1-\alpha)\left[(2 p+3)-(p+2) e^{\frac{1}{p+2}}\right]-\left[2\left(e^{\frac{1}{p+2}}-1\right)(p+2)+e^{\frac{1}{p+2}}\right]>0$

then $W_{\lambda, p+1}^{(1)}(z)$ is close-to-convex function order $\alpha \in[0,1)$ with respect to $\bar{J}_{p}(z)$ in $U$.

By taking $\alpha=0$ in Corollary 3.5 and using relationship $K(0)=K$, we obtain the following corollary.

Corollary 3.6. If $\lambda \geq 1$ and $p>x_{2}-2$, where $x_{2} \cong 5.4904$ is the root of the equation

$$
4 x-(3 x+1) e^{\frac{1}{x}}-1=0,
$$

then $W_{\lambda, p+1}^{(1)}(z)$ is close-to-convex function with respect to $\bar{J}_{p}(z)$ in $U$.

Proof. Let $\omega(x)=4 x-(3 x+1) e^{1 / x}-1, x>0$. By simple computation, we obtain

$$
\omega^{\prime}(x)=4-\frac{3 x^{2}-3 x-1}{x^{2}} e^{\frac{1}{x}}
$$


We observe that $\omega^{\prime}(x)>0$ for each $x>0$. Thus, function $\varphi(x)$ is an increasing function. Hence, $4(p+2)-(3 p+7) e^{1 /(p+2)}-1>0$ for every $p>x_{2}-2$. Here, $x_{2} \cong 5.4904$ is the root of the equation

$$
4 x-(3 x+1) e^{\frac{1}{x}}-1=0 .
$$

Thus, the proof of Corollary 3.6 is complete.

\section{CONCLUDING REMARKS}

In this study, two kinds of normalization of the Wright function are investigated. These normalized Wright functions are examinedin the generalized class of the close-to-convex function class. In the study, several sufficient conditions for the normalized Wright functions are obtained. The results obtained in this study are better than the results of Prajapat (see [7, p. 208, Theorem 2.12]).

\section{REFERENCES}

[1] Wright EM. On the coefficients of power series having exponential singularities, Journal London Mathematics Society, Volume 8, 1933, pp.71-79.

[2] Gorenflo R, Luchko Yu, Mainardi F. Analytic properties and applications of Wright functions, Fractional Calculus \& Applied Analysis, Volume 2, No. 4, 1999, pp.383-414.

[3] Podlubny I. Fractional differential equations, San Diego: Academic Press, 1999.

[4] Samko SG, Kilbas AA, Marichev OI. Fractional integrals and derivatives: Theory and Applications, New York: Gordon and Breach, 1993.

[5] Mainardi F. (Ed. Carpinteri A and Mainardi F.) Fractional calculus: some basic problemsin continuum and statistical mechanics. In: Fractals and Fractional Calculus in Continuum Mechanics, Wen: Springer Verlag, 1971.

[6] Luchko Yu, Gorenflo R. Scale-invariant solutions of a partial differential equation of fractional order, Fractional Calculus \& Applied Analysis, Volume 1, No 1, 1998, pp. 6378.

[7] Prajapat JK. Certain geometric properties of the Wright function, Inegral Transforms and Special Functions, Volume 26, No. 3, 2015, pp. 203-212.

[8] Duren PL. Univalent Functions, Grundlehren der Mathematischen Wissenshaften, Bd. 259, New York: Springer-Verlag, 1983.

[9] Goodman AW. Univalent Functions, Volume I, Washington: Polygonal, 1983.

[10] Srivastava HM and Owa S. (Editors) Current Topics in Analytic Function Theory, Singapore: World Scientific, 1992

[11] Goodman AW. Univalent functions, Vols. 1-2, Tampa, FL: Mariner, 1983.

[12] Murugusundaramoorthy G, Vijaya K and Porwal S. Some inclusion results of certain subclass of analytic functions associated with Poisson distribution series. Hacettepe Journal of Mathematics and Statistics, DOI: 10.15672/HJMS201664513110. (In press).

\section{CV/ÖZGEÇMiș}

Nizami MUSTAFA; Assoc. Prof. (Doç. Dr.)

He got his bachelors' and master's degree in the Faculty of Mechanics and Mathematics at Azerbaijan State University, Baku/Azerbaijan in 1981, PhD degree in the Faculty of Mechanics and Mathematics at Azerbaijan State University, Baku/Azerbaijan in 1991. Currently, he is an academic member of the Mathematical 
Department at Kafkas University. His major areas of interests are: Mathematical Analysis, Singular Integral Equations, Special Function Theory.

Lisans ve Yüksek Lisans derecesini 1981'de Azerbaycan Devlet Üniversitesi Matematik ve Mekanik Fakültesi'nden, Doktora derecesini 1991 yılında aynı Fakülte'nin Analiz ve Fonksiyonlar Teorisi Kürsüsü'nden aldr. Şu anda Kafkas Üniversitesi Fen-Edebiyat Fakültesi Matematik Bölümü'nde ögrretim üyesi olarak görev yapmaktadır. Temel çalışma alanları: Matematiksel Analiz, Singüler Integral Denklemler, Özel Fonksiyonlar Teorisi üzerinedir. 